\title{
Circadian Rhythms in Bacterial Sepsis Pathology: What We Know and What We Should Know
}

\author{
Malena Lis Mul Fedele ${ }^{1}$, Camila Agustina Senna ${ }^{2}$, Ignacio Aiello ${ }^{2}$, \\ Diego Andres Golombek ${ }^{2}$ and Natalia Paladino ${ }^{2 *}$
}

${ }^{1}$ Laboratorio de Cronofisiología, Instituto de Investigaciones Biomédicas/Pontificia Universidad Católica Argentina - Consejo Nacional de Investigaciones Científicas y Técnicas (UCA-CONICET), Buenos Aires, Argentina, ${ }^{2}$ Laboratorio de Cronobiología, Departamento de Ciencia y Tecnología, Universidad Nacional de Quilmes/Consejo Nacional de Investigaciones Cientificas y Técnicas (CONICET), Buenos Aires, Argentina

\section{OPEN ACCESS}

Edited by: Isaac Kirubakaran Sundar, University of Kansas Medical Center, United States

Reviewed by: Brian James Altman, University of Rochester, United States Lauren Hartstein, University of Colorado Boulder, United States

*Correspondence: Natalia Paladino napaladino76@gmail.com

Specialty section: This article was submitted to Bacteria and Host a section of the journal Frontiers in Cellular and Infection Microbiology

Received: 09 September 2021 Accepted: 23 November 2021 Published: 09 December 2021

Citation: Mul Fedele ML, Senna CA, Aiello I, Golombek DA and Paladino N (2021)

Circadian Rhythms in Bacterial Sepsis Pathology: What We Know and What We Should Know. Front. Cell. Infect. Microbiol. 11:773181. doi: 10.3389/fcimb.2021.773181
Sepsis is a syndrome caused by a deregulated host response to infection, representing the primary cause of death from infection. In animal models, the mortality rate is strongly dependent on the time of sepsis induction, suggesting a main role of the circadian system. In patients undergoing sepsis, deregulated circadian rhythms have also been reported. Here we review data related to the timing of sepsis induction to further understand the different outcomes observed both in patients and in animal models. The magnitude of immune activation as well as the hypothermic response correlated with the time of the worst prognosis. The different outcomes seem to be dependent on the expression of the clock gene Bmal1 in the liver and in myeloid immune cells. The understanding of the role of the circadian system in sepsis pathology could be an important tool to improve patient therapies.

Keywords: sepsis, circadian rhythms, immune system, hypothermia, infection

\section{INTRODUCTION}

\section{Sepsis and Septic Shock Characteristics}

Sepsis is a syndrome characterized by multi-organ dysfunction caused by a deregulated host response to a pathogen, and is the primary cause of death from infection (Singer, 2016) affecting more than 30 million people worldwide every year with an in-hospital mortality rate of about $25-40 \%$ (Vincent et al., 2014; Acuna-Castroviejo et al., 2017; Huang et al., 2019). Sepsis symptoms vary according to the site of infection, the type of invasive microorganism, previous patient health and the time of detection; and include: body temperature alterations (fever or hypothermia), elevated heart and respiratory rate, altered mental state, glucose metabolism, inflammatory and hemodynamic variables, among others (Angus and van der Poll, 2013). Septic shock is defined as the septic condition worsened by metabolic and circulatory alterations, as hypotension, which increases the mortality rate (Singer, 2016). Patients who survive can present long-term physical, psychological, and cognitive disorders (Huang et al., 2019). Despite the severity of this pathology, there is no effective therapy that significantly reduces mortality and morbidity (Acuna-Fernandez et al., 2020).

Pathogen infection triggers an important inflammatory response, inducing an increase of proinflammatory cytokine release, such as IL- 6 , IL-1 $\beta$ and TNF- $\alpha$, known as "cytokine storm", generating a systemic inflammatory response [SIRS (Chen et al., 2014)]. The inflammatory response is not limited to the site of infection. The increased levels of IL-6 induce the production of the $\mathrm{C}$-reactive protein in the liver, an acute response protein with anticoagulant and antiapoptotic 
function (among others), that can be used as a potential septicemia biomarker predictor (Cinel and Opal, 2009). Furthermore, an anti-inflammatory response, including glucocorticoid (Marik, 2011), IL-10, IL-1 receptor antagonist (IL-1Ra) and TGF- $\beta$ secretion (Marchant et al., 1994; Marie et al., 1996), is also elicited. An excess of inflammation can cause tissue damage while an excess of anti-inflammatory response can favor secondary infections (van der Poll and Opal, 2008). The levels of IL-10 correlate with a worst patient prognosis (Gogos et al., 2000).

As in other pathologies, the central nervous system participates in sepsis development, as evidenced by the increase in proinflammatory molecule levels in this tissue (Meneses et al., 2019). Vagus nerve activation triggers the production of acetylcholine (one of the main vagus neurotransmitters), which inhibits synthesis and release of immune mediators by macrophages, such as TNF- $\alpha$, increasing lifespan in septic mice (Borovikova et al., 2000; Andersson and Tracey, 2012). Moreover, the immune response activates the hypothalamic-pituitary-adrenal (HPA) axis which ends in glucocorticoids secretion, that modulates many body functions such as liver gluconeogenesis, glucose uptake, immune functions, among others (Kasahara and Inoue, 2015). Finally, the proinflammatory response can alter the blood-brain barrier (BBB), increasing its permeability (Danielski et al., 2018) and allowing the entrance of cytokines and leukocytes (Bohatschek et al., 2001; Hofer et al., 2008).

\section{Animal Models of Sepsis and Septic Shock} One of the most studied animal models of sepsis is induced by the administration of high doses (close to $20 \mathrm{mg} / \mathrm{kg}$ ) of the bacterial endotoxin lipopolysaccharide (LPS), inducing high mortality rates (Liao and Lin, 2015; Ramos-Benitez et al., 2018) and the characteristic signs of sepsis (Nautiyal et al., 2009; Chuaiphichai et al., 2016; Cordoba-Moreno et al., 2018; Ramos-Benitez et al., 2018). It is a simple and reproducible technique, whose dosedependent effects depend on the bacterial and mice strain used.

Administration of live bacteria is an easy, reproducible and low invasive method that can be used to study the response to a specific pathogen without surgery (Korneev, 2019). This technique can be useful to model hospital infections, which are often caused by monoinfections.

Cecal ligation and puncture (CLP) is considered a model of human appendix rupture or perforated diverticulitis. It is induced by midline laparotomy, exteriorization and ligation of the caecum, and puncture of the ligated caecum. The severity of the response can be adjusted by the size and number of punctions (Buras et al., 2005).

\section{CIRCADIAN MODULATION OF SEPTIC PATHOLOGY}

\section{Circadian Variations in Animal Models of Sepsis}

There is a daily variation in the mortality rate due to septic shock: mice injected intraperitoneally with high doses of LPS at the end of their resting phase of activity (i.e., the end of the day) show a higher mortality rate ( $80 \%$ approximately) than those injected in the middle of the active phase of activity [the middle of the night, 30\% approximately (Halberg et al., 1960; Marpegan et al., 2009)]. Similar results were obtained when TNF- $\alpha$ was administered intravenously (Hrushesky et al., 1994). Moreover, the clearance of Salmonella enterica was higher if the infection occurred during the active phase [the night (Bellet et al., 2013)]. Additionally, sepsis induced by CLP has a worse outcome when surgery is performed at the end of the active phase (the night) in comparison with the middle of the rest phase [the day (Heipertz et al., 2018)]. The differences in the time of poor prognosis between models could be related with differences in the kinetics of each stimulation method.

The relationship between sepsis and the molecular circadian clock machinery has also been studied. The molecular mechanism of the circadian clock arises from negative transcriptional feedback loops. The core loop includes the positive elements Clock and Bmal1, inducing the expression of the negative elements Per1-3 and Cry1-2, which, in turn, repress the transcriptional activity of the positive elements generating oscillations with periods close to 24 hours (Partch et al., 2014). It was shown that mice deficient for Per2 (Per2 KO) are more resistant to LPS-induced septic shock, showing lower levels of IFN- $\gamma$, IL-1 $\beta$ (Liu et al., 2006) and higher levels of glucocorticoids (Wang et al., 2015). There are many studies showing that the oscillation of circadian clock genes is disrupted in these mice (Zheng et al., 1999; Liu et al., 2006). Moreover, they also exhibited higher mRNA expression of the clock genes Clock and Bmall and of the enzyme Star, which participates in the adrenal synthesis of glucocorticoids, after LPS administration (Wang et al., 2015). Similarly, Clock-deficient mice showed greater survival after CLP-induced sepsis. Additionally, both mutants lost the daily difference in the mortality rate (Liu et al., 2006; Wang et al., 2016; Heipertz et al., 2018). Thus, it was suggested that the adrenal peripheral clock may play an important role in modulating the timing of LPS-corticoid secretion via regulation of the enzyme Star (Wang et al., 2015). Collectively, these data suggest that the daily differences observed in the mortality rate depend on the functioning of the clock rather than a specific clock component, since Per2 KO mice showed higher levels of Clock and both mutant animals were more resistant to sepsis than WT mice.

\section{Immune-Mediated Circadian Regulation}

We recently reported that LPS-induced sepsis at the end of the resting phase elicited higher levels of TNF- $\alpha$ in serum than animals inoculated at the active phase (Mul Fedele et al., 2020). In addition, TNFR1-deficient mice not only had higher survival [which was previously observed by (Pfeffer et al., 1993; Leon et al., 1998; Guo et al., 2009)], but also abolished the daily difference. Additionally, it was observed that circadian variation in the mortality rate was abolished in mice lacking Toll-Like Receptor (TLR)-2 in sepsis induced via CLP (Heipertz et al., 2018).

Macrophages have an essential role during all sepsis stages (Freudenberg et al., 1986; Cheng et al., 2018). Peritoneal macrophages, the first ones to be activated after intraperitoneal 
stimulation, can be classified, according to their phenotype and function, in large and small cells, LPMs and SPMs, respectively. SPMs and LPMs exhibit specialized functions: SPMs present a pro-inflammatory functional profile, and LPMs appear to have a role in the maintenance of peritoneal cavity physiological conditions. LPMs are the most abundant subset of macrophages in unstimulated conditions, whereas SPMs are the minor subset. Nevertheless, in response to infectious or inflammatory stimuli, the cellular composition of the peritoneal cavity is altered (Cassado Ados et al., 2015). Despite the relatively little information focused on the functional profile induced by stimulation of SPM and LPM cells, data showed that both cell types can differentiate into both M1 (classically activated, pro-inflammatory) and M2 (alternative activated, anti-inflammatory) cells depending on the stimuli (Yuan et al., 2017; Li et al., 2021). Moreover, immune stimuli induce a decrease in LPMs levels, a phenomenon known as macrophage disappearance reaction [MDR (Okabe and Medzhitov, 2014)], which can include cell apoptosis (Luan et al., 2015), local clot form (Zhang et al., 2019) and migration (Okabe and Medzhitov, 2014). These cells accumulate in the omentum and can interact with mesothelial cells (Okabe and Medzhitov, 2014). Interestingly, this phenomenon was more pronounced after LPS administration at the time of higher mortality [at the end of the resting phase (Mul Fedele et al., 2020)]. In response to a sterile injury in the liver, LPMs invaded afflicted tissue via direct recruitment across the mesothelium (Wang and Kubes, 2016). Regarding this, it was recently found that conditional deletion of Bmall in hepatocytes (Bmal1 ${ }^{\Delta \mathrm{Hep}}$ ) results in constitutively high LPS sensitivity (Geiger et al., 2021) suggesting that liver signals could induce the arrival of LPMs in a clock-dependent way.

The decrease in the levels of LPMs is accompanied by the arrival of inflammatory monocytes (Cassado Ados et al., 2015) which can differentiate into SPMs (Ghosn et al., 2010), which increase 2 days after LPS stimulation. These cells showed higher levels at the end of the resting phase (Mul Fedele et al., 2020). In vitro studies have shown that SPMs develop a proinflammatory profile in response to LPS (Cain et al., 2013) suggesting a relation with the higher levels of TNF- $\alpha$ observed at this time (Mul Fedele et al., 2020).

Spleen macrophages are also activated (measured by CD86 expression) in response to LPS (Liu et al., 2006). Interestingly, this activation increases after LPS administration during the resting phase, but not in the active phase (Mul Fedele et al., 2020).

Recent works showed that conditional deletion of Bmal1 in myeloid cells (Bmal1 ${ }^{\Delta \mathrm{Mye}}$ ), which disrupt the macrophage clock, accelerated death in sepsis induced by both CLP (Deng et al., 2018) or LPS (Curtis et al., 2015) and abolished the daily differences in the mortality rate. Bmal1 ${ }^{\Delta \text { Mye }}$ mice developed higher pro-inflammatory (Curtis et al., 2015) and antiinflammatory responses (Deng et al., 2018). The interaction between the pathogen and the host immune system is very complex and comprises two stages: an early stage characterized by excessive inflammation and a late stage characterized by sustained immune suppression (van der Poll et al., 2017).
Therefore, it is not surprising to find that Bmall is related to pro-inflammatory and anti-inflammatory processes, which are both deregulated during sepsis. In contrast, daily differences after LPS inoculation were not abolished in Bmal1 ${ }^{\Delta \mathrm{Mye}}$ mice maintained under a time-restricted feeding schedule (Geiger et al., 2021). This discrepancy could be due to the different kinetics observed in the sepsis-induced mortality between models since the survival time was shorter in the last mentioned study. In addition, it is possible that mice fed only during the night have more robust peripheral circadian rhythms that may compensate for the lack of Bmal1 in myeloid cells, similar to what happens with the rhythm of the respiratory exchange rate in the same work (Geiger et al., 2021).

Regarding anti-inflammatory mechanisms, the serum levels of IL-10 and corticosterone increased after LPS injection, without time differences (Mul Fedele et al., 2020; Geiger et al., 2021). It has been shown that, in animal models, the stimulation of the HPA axis (which is responsible for glucocorticoid secretion) increases resistance to the endotoxic shock (Dejager et al., 2010; Gilibert et al., 2014). However, clinical therapy with glucocorticoids does not always induce longer survival and can generate treatment resistance (Dendoncker and Libert, 2017). Additionally, melatonin, which participates in circadian rhythm regulation, has anti-inflammatory properties and protects against sepsis-induced cardiac (Zhang et al., 2019), lung (Li et al., 2020), liver (Chen et al., 2019) and renal dysfunction (Dai et al., 2019). These effects could be dependent on melatonin receptor-induced neutrophil activity (Xu et al., 2019).

This evidence may indicate that circadian control over some physiological functions, such as the immune system, that are altered during sepsis, can have an important role on the pathogenesis of this syndrome.

\section{Circadian Regulation of Body Temperature During Sepsis}

As previously mentioned, another feature of sepsis is the alteration of body temperature: fever or hypothermia, the latter being associated with a worse prognosis (Angus and van der Poll, 2013) and higher inflammation (Nautiyal et al., 2009; Stewart et al., 2010; Garami et al., 2018). Interestingly, in both CLP (Silver et al., 2012) and LPS-induced sepsis (Mul Fedele et al., 2020) deeper hypothermia correlated with higher mortality (the active phase and the end of the resting phase, respectively).

The hypothalamic preoptic area (POA) is the main integrative brain site for thermoregulation (Morrison and Nakamura, 2018), but also the suprachiasmatic (SCN) and paraventricular (PVN) nucleus modulate thermal signals (Wanner et al., 2013; Guzman-Ruiz et al., 2015). LPS inoculation induces neuronal activation of these brain regions (Hare et al., 1995; Marpegan et al., 2005; Paladino et al., 2014). Interestingly, this activation (measured by cFOS expression) was increased after LPS inoculation at the time of higher mortality rate [the end of the resting phase (Mul Fedele et al., 2020)]. TNF$\alpha$ and TNFR1 participate both in the immune-circadian communication (Cavadini et al., 2007; Duhart et al., 2013; Paladino et al., 2014) and in the hypothermic response to LPS 
(Leon et al., 1998; Nautiyal et al., 2009). We have shown that these molecules were induced in the POA in response to septic shock, although it was independent of the time of LPS inoculation (Mul Fedele et al., 2020).

Temperature is a well-known circadian entraining cue for peripheral oscillators. It was shown that circadian changes in temperature, similar to that seen in core temperature rhythms, can entrain and enhance the amplitude of circadian rhythms in the periphery (Buhr et al., 2010). In addition, the circadian clock is responsible for the development of a body temperature circadian rhythm (Refinetti and Menaker, 1992), so that this bidirectional interaction is disrupted during sepsis.

\section{Role of the Circadian System in Metabolic and Circulatory Alterations}

There are many interactions between metabolic or circulatory functions and the clock machinery. As previously mentioned, septic patients can develop hypoglycemia through the induction of glycolysis, which could be lethal if it is not compensated with liver gluconeogenesis (Weis et al., 2017). Using a time-restricted feeding protocol, the LPS-induced hypoglycemia correlated with higher mortality rate [the end of the resting phase (Geiger et al., 2021)], while $\beta$-hydroxybutyrate levels, which induces NLRP3 inflammasome (Youm et al., 2015), are increased at this time. This inflammasome, along with reactive oxygen and nitrogen species and $N F-\kappa B$ pathway can be regulated in the mitochondria both by clock genes and melatonin [reviewed in (Acuna-Castroviejo et al., 2017)].

The circadian clock also regulates coagulation and fibrinolysis processes (Budkowska et al., 2019). Bmal1-deficient mice exhibited a hypercoagulable state and an enhanced arterial and venous thrombogenicity (Hemmeryckx et al., 2019). Moreover, the lack of Bmal1 induces alterations in the levels of coagulation factors, contributing to a coagulation abnormality in $S$. oralis infection (Chen et al., 2020). In addition, tissue factor (TF), which participates in coagulation and in LPS-induced inflammation and mortality, is under circadian regulation in the liver (Oishi et al., 2013).

In spite of these data, it remains to be explored what happens with these mechanisms when sepsis is induced at different times of the day and what happens with their circadian rhythms during the pathology.

\section{Sepsis and Circadian Desynchronization}

Desynchronization of circadian rhythms achieved by different protocols (SCN injury or chronic jet-lag models) increased LPSinduced inflammation (Castanon-Cervantes et al., 2010; Adams et al., 2013; Guerrero-Vargas et al., 2014), while constant light conditions (also disruptive) reduced the survival rate after CLP (Carlson and Chiu, 2008). Interestingly, the exacerbation in the mortality rate and hypothermic response is accompanied by an increase in the TNF- $\alpha$ levels in desynchronized mice (Mul Fedele et al., 2020). Similarly, four inversions of the LD cycle (1 per week) increased the mortality rate (Castanon-Cervantes et al., 2010). Moreover, other studies from our group have shown that in constant dark conditions, the daily difference in the response to LPS is lost with low survival percentages at all times
(Marpegan et al., 2009). This suggests that the absence of temporal external cues as well as desynchronization can reduce the survival percentage in sepsis. This data also supports the bidirectional communication between the immune system and the circadian system (Marpegan et al., 2009) and suggests a strong influence of the central clock on susceptibility to immune stimuli.

Additionally, the uncoupling of central and peripheral clocks induced by reversed feeding (feeding only at the resting phase) also increased the mortality rate and exacerbated the inflammatory response, compared with active phase-fed mice, after CLP (Oyama et al., 2014). Interestingly, reversed feeding inverted the time of higher mortality induced by LPS inoculation (Geiger et al., 2021). In addition Bmal1 ${ }^{\Delta \mathrm{Hep}}$ mice developed constitutively high LPS sensitivity independently from light or feeding schedules, in a farnesoid X receptor (FXR)-dependent way (Geiger et al., 2021).

\section{Influence of the Circadian System in Patient Response}

In relation to patients, it has been shown that Salmonella abortus inoculation at the end of the active phase (the day) induces higher levels of serum cortisol, compared to its administration at the beginning of the active phase [the day (Pollmacher et al., 1996)]. In addition, a day-night difference in the acute phase response to endotoxemia exists in healthy volunteers with a more pronounced inflammatory response during the resting phase [the night (Alamili et al., 2014)]. Moreover, septic patients had higher levels of cytokines than other intensive care unit (ICU) patients (Acuna-Fernandez et al., 2020) and exhibited several alterations in clock gene rhythms (Coiffard et al., 2019; Acuna-Fernandez et al., 2020; Lachmann et al., 2021). These alterations were accompanied by an increase in urinary 6-sulfatoxymelatonin (aMT6s) excretion, which is related to chronodisruption (Acuna-Fernandez et al., 2020). A longitudinal study reported that individuals who developed septic shock during their ICU stay had an increase in aMT6s and a decrease in cortisol levels compared with entry and discharge from the ICU. Also, higher aMT6s or cortisol mean values were correlated with lower inhospital mortality (Sertaridou et al., 2018). Studies carried out in patients who suffered severe trauma showed that the early alteration of the cortisol rhythms are associated with sepsis development. These parameters were accompanied by clock gene and leucocyte count alterations (Coiffard et al., 2019). However, changes in the rhythms of clock gene expression and rest-activity were also observed in non-septic ICU patients (Maas et al., 2020a; Maas et al., 2020b). Since sleep deprivation increases inflammation (Shearer et al., 2001; Frey et al., 2007), it is relevant to consider that ICU normally have constant light conditions, which can complicate the inflammatory response.

Because of the correlation between sepsis and circadian rhythms, chronobiological therapies have been proposed. As septic patients have shown alterations in circadian rhythms and sleep in ICU, a few studies used light therapy to entrain circadian rhythms. In septic animals, exposure to bright blue light enhanced bacterial clearance, reduced systemic inflammation and organ injury (Lewis et al., 2018). However, light therapy did not 
improve patient prognosis, although these works used low intensity light [reviewed in (Jacob et al., 2020)]. As previously mentioned, melatonin has shown anti-inflammatory properties in sepsis (Marra et al., 2019; Jacob et al., 2020). In addition, melatonin reduced IL-1 $\beta$ and YKL-40 plasma levels and oxidative stress response in a human model of endotoxemia during the active phase, but not during the resting phase (Alamili et al., 2014).

\section{CONCLUSION}

Despite the numerous evidences that show a circadian component of the septic response, the causes underlying this differential response are still unknown. Figure 1 summarizes the main findings that could explain the different outcomes after sepsis induction at different times. Immune stimuli induce higher cytokine secretion at the time of higher mortality (the end of the resting phase), which could be secreted by SPMs or splenic macrophages, among other cells. In addition, the MDR is higher at the time of worse prognosis (the end of the resting phase). Circadian rhythms in the liver could be relevant for the increase in glucose metabolism alterations and acute phase protein secretion, including pro-inflammatory cytokines, at the moment of higher mortality. In addition, activation of brain regions that control body temperature, and the subsequent hypothermia, were also associated with poor prognosis (the end of the resting phase). Lastly, the levels of glucocorticoids and IL10, both anti-inflammatory mediators, increase similarly at different times. The nervous system, particularly the HPA axis and autonomic nerves, regulates many of these functions. To further understand the causes of the daily differences in sepsis prognosis it will be relevant to deepen the knowledge of the mechanisms inducing a higher pro-inflammatory immune



FIGURE 1 | Differential features observed at times of higher and lower mortality rates. Infectious stimuli impact the peritoneal macrophages (SPMs and LPMs), in the liver and the spleen, secreting higher cytokine levels to the blood at the time of higher mortality. This difference could be related to the higher basal SPMs counts and higher activated (CD86) splenic macrophages after stimulation. The decrease in the LPMs count (MDR) is also higher at the time of the worst prognosis. The antiinflammatory mediators glucocorticoids and IL10 increase similarly at both times. The decrease in glucose and the increase in $\beta$-hydroxybutyrate (BHB) levels were more pronounced at the moment of higher mortality and depending on the liver rhythms. Neuronal activation (cFos) of brain regions that control body temperature (POA, SCN and PVN), and the subsequent hypothermia, were also increased at the time of poor prognosis. Communication between the central nervous system and peripheral tissues during sepsis includes the HPA axis and the autonomic nerves, particularly the vagus nerve. The brain blood barrier (BBB) can receive inflammatory signals and secrete cytokines into the brain. In addition, the inflammation that occurs during sepsis can disturb this barrier and increase its permeability. The expression of the clock gene Bmal1 in the liver and the myeloid cells is associated with a better prognosis. 
response at specific times and, also, to analyze the role of the nervous system and the liver at each time of day.

The understanding of the role of the circadian clock in patients in the ICU, particularly after infections or severe trauma, could help to improve sepsis treatments. In addition, circadian evaluation could be used as a prognosis marker. In this regard, it will be useful to measure rhythmic variables, like immune cell count, melatonin or cortisol, at different times of the day, during the early stage of the disease in order to associate this with patient prognosis. Likewise, analyzing the time of infection, for example in individuals who suffered severe trauma, would allow us to assess whether the circadian differences observed in animal models can be extrapolated to humans.

\section{REFERENCES}

Acuna-Castroviejo, D., Rahim, I., Acuna-Fernandez, C., Fernandez-Ortiz, M., Solera-Marin, J., Sayed, R. K. A., et al. (2017). Melatonin, Clock Genes and Mitochondria in Sepsis. Cell Mol. Life Sci. 74 (21), 3965-3987. doi: 10.1007/ s00018-017-2610-1

Acuna-Fernandez, C., Marin, J. S., Diaz-Casado, M. E., Rusanova, I., DariasDelbey, B., Perez-Guillama, L., et al. (2020). Daily Changes in the Expression of Clock Genes in Sepsis and Their Relation With Sepsis Outcome and Urinary Excretion of 6-Sulfatoximelatonin. Shock 53 (5), 550-559. doi: 10.1097/ SHK.0000000000001433

Adams, K. L., Castanon-Cervantes, O., Evans, J. A., and Davidson, A. J. (2013). Environmental Circadian Disruption Elevates the IL-6 Response to Lipopolysaccharide in Blood. J. Biol. Rhythms 28 (4), 272-277. doi: 10.1177/ 0748730413494561

Alamili, M., Bendtzen, K., Lykkesfeldt, J., Rosenberg, J., and Gogenur, I. (2014). Pronounced Inflammatory Response to Endotoxaemia During Nighttime: A Randomised Cross-Over Trial. PLoS One 9 (1), e87413. doi: 10.1371/ journal.pone.0087413

Andersson, U., and Tracey, K. J. (2012). Neural Reflexes in Inflammation and Immunity. J. Exp. Med. 209 (6), 1057-1068. doi: 10.1084/jem.20120571

Angus, D. C., and van der Poll, T. (2013). Severe Sepsis and Septic Shock. N. Engl. J. Med. 369 (9), 840-851. doi: 10.1056/NEJMra1208623

Bellet, M. M., Deriu, E., Liu, J. Z., Grimaldi, B., Blaschitz, C., Zeller, M., et al. (2013). Circadian Clock Regulates the Host Response to Salmonella. Proc. Natl. Acad. Sci. U. S. A. 110 (24), 9897-9902. doi: 10.1073/pnas.1120636110

Bohatschek, M., Werner, A., and Raivich, G. (2001). Systemic LPS Injection Leads to Granulocyte Influx Into Normal and Injured Brain: Effects of ICAM-1 Deficiency. Exp. Neurol. 172 (1), 137-152. doi: 10.1006/exnr.2001.7764

Borovikova, L. V., Ivanova, S., Nardi, D., Zhang, M., Yang, H., Ombrellino, M., et al. (2000). Role of Vagus Nerve Signaling in CNI-1493-Mediated Suppression of Acute Inflammation. Auton. Neurosci. 85 (1-3), 141-147. doi: 10.1016/S1566-0702(00)00233-2

Budkowska, M., Lebiecka, A., Marcinowska, Z., Wozniak, J., Jastrzebska, M., and Dolegowska, B. (2019). The Circadian Rhythm of Selected Parameters of the Hemostasis System in Healthy People. Thromb. Res. 182, 79-88. doi: 10.1016/ j.thromres.2019.08.015

Buhr, E. D., Yoo, S. H., and Takahashi, J. S. (2010). Temperature as a Universal Resetting Cue for Mammalian Circadian Oscillators. Science 330, 379-385. doi: 10.1126/science.1195262

Buras, J. A., Holzmann, B., and Sitkovsky, M. (2005). Animal Models of Sepsis: Setting the Stage. Nat. Rev. Drug Discov. 4 (10), 854-865. doi: 10.1038/nrd1854

Cain, D. W., O'Koren, E. G., Kan, M. J., Womble, M., Sempowski, G. D., Hopper, K., et al. (2013). Identification of a Tissue-Specific, C/EBPbeta-Dependent Pathway of Differentiation for Murine Peritoneal Macrophages. J. Immunol. 191 (9), 4665-4675. doi: 10.4049/jimmunol.1300581

Carlson, D. E., and Chiu, W. C. (2008). The Absence of Circadian Cues During Recovery From Sepsis Modifies Pituitary-Adrenocortical Function and Impairs Survival. Shock 29 (1), 127-132. doi: 10.1097/shk.0b013e318142c5a2

\section{AUTHOR CONTRIBUTIONS}

MM and NP initiated and designed the overall concept and wrote the manuscript. CS and IA wrote the manuscript. All authors revised the manuscript, approved the final version and approved it for publication.

\section{FUNDING}

This work was supported by grants from the National Research Council (CONICET), and the National University of Quilmes (UNQ).

Cassado Ados, A., D'Imperio Lima, M. R., and Bortoluci, K. R. (2015). Revisiting Mouse Peritoneal Macrophages: Heterogeneity, Development, and Function. Front. Immunol. 6, 225. doi: 10.3389/fimmu.2015.00225

Castanon-Cervantes, O., Wu, M., Ehlen, J. C., Paul, K., Gamble, K. L., Johnson, R. L., et al. (2010). Dysregulation of Inflammatory Responses by Chronic Circadian Disruption. J. Immunol. 185 (10), 5796-5805. doi: 10.4049/ jimmunol.1001026

Cavadini, G., Petrzilka, S., Kohler, P., Jud, C., Tobler, I., Birchler, T., et al. (2007). TNF-Alpha Suppresses the Expression of Clock Genes by Interfering With EBox-Mediated Transcription. Proc. Natl. Acad. Sci. U. S. A. 104 (31), $12843-$ 12848. doi: 10.1073/pnas.0701466104

Cheng, Y., Marion, T. N., Cao, X., Wang, W., and Cao, Y. (2018). Park 7: A Novel Therapeutic Target for Macrophages in Sepsis-Induced Immunosuppression. Front. Immunol. 9, 2632. doi: 10.3389/fimmu.2018.02632

Chen, L., Li, S., Nie, J., Zhao, J., Yu, S., Li, Y., et al. (2020). Bmall Regulates Coagulation Factor Biosynthesis in Mouse Liver in Streptococcus Oralis Infection. Front. Cell Infect. Microbiol. 10, 530190. doi: 10.3389/fcimb. 2020.530190

Chen, P., Stanojcic, M., and Jeschke, M. G. (2014). Differences Between Murine and Human Sepsis. Surg. Clin. North Am. 94 (6), 1135-1149. doi: 10.1016/ j.suc.2014.08.001

Chen, J., Xia, H., Zhang, L., Zhang, H., Wang, D., and Tao, X. (2019). Protective Effects of Melatonin on Sepsis-Induced Liver Injury and Dysregulation of Gluconeogenesis in Rats Through Activating SIRT1/STAT3 Pathway. BioMed. Pharmacother. 117, 109150. doi: 10.1016/j.biopha.2019.109150

Chuaiphichai, S., Starr, A., Nandi, M., Channon, K. M., and McNeill, E. (2016). Endothelial Cell Tetrahydrobiopterin Deficiency Attenuates LPS-Induced Vascular Dysfunction and Hypotension. Vascul. Pharmacol. 77, 69-79. doi: 10.1016/j.vph.2015.08.009

Cinel, I., and Opal, S. M. (2009). Molecular Biology of Inflammation and Sepsis: A Primer. Crit. Care Med. 37 (1), 291-304. doi: 10.1097/CCM.0b013e31819267fb

Coiffard, B., Diallo, A. B., Culver, A., Mezouar, S., Hammad, E., Vigne, C., et al. (2019). Circadian Rhythm Disruption and Sepsis in Severe Trauma Patients. Shock 52 (1), 29-36. doi: 10.1097/SHK.0000000000001241

Cordoba-Moreno, M. O., Todero, M. F., Fontanals, A., Pineda, G., Daniela, M., Yokobori, N., et al. (2018). Consequences of the Lack of IL-10 in Different Endotoxin Effects and Its Relationship With Glucocorticoids. Shock 52 (2), 264-273. doi: 10.1097/SHK.0000000000001233

Curtis, A. M., Fagundes, C. T., Yang, G., Palsson-McDermott, E. M., Wochal, P., McGettrick, A. F., et al. (2015). Circadian Control of Innate Immunity in Macrophages by miR-155 Targeting Bmall. Proc. Natl. Acad. Sci. U. S. A. 112 (23), 7231-7236. doi: 10.1073/pnas.1501327112

Dai, W., Huang, H., Si, L., Hu, S., Zhou, L., Xu, L., et al. (2019). Melatonin Prevents Sepsis-Induced Renal Injury via the PINK1/Parkin1 Signaling Pathway. Int. J. Mol. Med. 44 (4), 1197-1204. doi: 10.3892/ijmm.2019.4306

Danielski, L. G., Giustina, A. D., Badawy, M., Barichello, T., Quevedo, J., DalPizzol, F., et al. (2018). Brain Barrier Breakdown as a Cause and Consequence of Neuroinflammation in Sepsis. Mol. Neurobiol. 55 (2), 1045-1053. doi: $10.1007 /$ s12035-016-0356-7 
Dejager, L., Pinheiro, I., Puimege, L., Fan, Y. D., Gremeaux, L., Vankelecom, H., et al. (2010). Increased Glucocorticoid Receptor Expression and Activity Mediate the LPS Resistance of SPRET/EI Mice. J. Biol. Chem. 285 (40), 31073-31086. doi: 10.1074/jbc.M110.154484

Dendoncker, K., and Libert, C. (2017). Glucocorticoid Resistance as a Major Drive in Sepsis Pathology. Cytokine Growth Factor Rev. 35, 85-96. doi: 10.1016/ j.cytogfr.2017.04.002

Deng, W., Zhu, S., Zeng, L., Liu, J., Kang, R., Yang, M., et al. (2018). The Circadian Clock Controls Immune Checkpoint Pathway in Sepsis. Cell Rep. 24 (2), 366378. doi: 10.1016/j.celrep.2018.06.026

Duhart, J. M., Leone, M. J., Paladino, N., Evans, J. A., Castanon-Cervantes, O., Davidson, A. J., et al. (2013). Suprachiasmatic Astrocytes Modulate the Circadian Clock in Response to TNF-Alpha. J. Immunol. 191 (9), 46564664. doi: 10.4049/jimmunol.1300450

Freudenberg, M. A., Keppler, D., and Galanos, C. (1986). Requirement for Lipopolysaccharide-Responsive Macrophages in Galactosamine-Induced Sensitization to Endotoxin. Infect. Immun. 51 (3), 891-895. doi: 10.1128/ iai.51.3.891-895.1986

Frey, D. J., Fleshner, M., and Wright, K. P.Jr. (2007). The Effects of 40 Hours of Total Sleep Deprivation on Inflammatory Markers in Healthy Young Adults. Brain Behav. Immun. 21 (8), 1050-1057. doi: 10.1016/j.bbi.2007.04.003

Garami, A., Steiner, A. A., and Romanovsky, A. A. (2018). Fever and Hypothermia in Systemic Inflammation. Handb. Clin. Neurol. 157, 565-597. doi: 10.1016/ B978-0-444-64074-1.00034-3

Geiger, S. S., Traba, J., Richoz, N., Farley, T. K., Brooks, S. R., Petermann, F., et al. (2021). Feeding-Induced Resistance to Acute Lethal Sepsis Is Dependent on Hepatic BMAL1 and FXR Signalling. Nat. Commun. 12 (1), 2745. doi: 10.1038/ s41467-021-22961-Z

Ghosn, E. E., Cassado, A. A., Govoni, G. R., Fukuhara, T., Yang, Y., Monack, D. M., et al. (2010). Two Physically, Functionally, and Developmentally Distinct Peritoneal Macrophage Subsets. Proc. Natl. Acad. Sci. U. S. A. 107 (6), 25682573. doi: $10.1073 /$ pnas. 0915000107

Gilibert, S., Galle-Treger, L., Moreau, M., Saint-Charles, F., Costa, S., Ballaire, R., et al. (2014). Adrenocortical Scavenger Receptor Class B Type I Deficiency Exacerbates Endotoxic Shock and Precipitates Sepsis-Induced Mortality in Mice. J. Immunol. 193 (2), 817-826. doi: 10.4049/jimmunol.1303164

Gogos, C. A., Drosou, E., Bassaris, H. P., and Skoutelis, A. (2000). Pro- Versus Anti-Inflammatory Cytokine Profile in Patients With Severe Sepsis: A Marker for Prognosis and Future Therapeutic Options. J. Infect. Dis. 181 (1), 176-180. doi: $10.1086 / 315214$

Guerrero-Vargas, N. N., Salgado-Delgado, R., Basualdo Mdel, C., Garcia, J., Guzman-Ruiz, M., Carrero, J. C., et al. (2014). Reciprocal Interaction Between the Suprachiasmatic Nucleus and the Immune System Tunes Down the Inflammatory Response to Lipopolysaccharide. J. Neuroimmunol. 273 (12), 22-30. doi: 10.1016/j.jneuroim.2014.05.012

Guo, Z., Wang, S., Jiao, Q., Xu, M., and Xu, Z. (2009). Soluble TNFR II/IgG1 Fc Fusion Protein Treatment in the LPS-Mediated Septic Shock of Rats. BioMed. Pharmacother. 63 (7), 537-542. doi: 10.1016/j.biopha.2008.08.012

Guzman-Ruiz, M. A., Ramirez-Corona, A., Guerrero-Vargas, N. N., Sabath, E., Ramirez-Plascencia, O. D., Fuentes-Romero, R., et al. (2015). Role of the Suprachiasmatic and Arcuate Nuclei in Diurnal Temperature Regulation in the Rat. J. Neurosci. 35 (46), 15419-15429. doi: 10.1523/JNEUROSCI.1449-15.2015

Halberg, F., Johnson, E. A., Brown, B. W., and Bittner, J. J. (1960). Susceptibility Rhythm to E. Coli Endotoxin and Bioassay. Proc. Soc. Exp. Biol. Med. 103, 142144. doi: 10.3181/00379727-103-25439

Hare, A. S., Clarke, G., and Tolchard, S. (1995). Bacterial LipopolysaccharideInduced Changes in FOS Protein Expression in the Rat Brain: Correlation With Thermoregulatory Changes and Plasma Corticosterone. J. Neuroendocrinol. 7 (10), 791-799. doi: 10.1111/j.1365-2826.1995.tb00716.x

Heipertz, E. L., Harper, J., Lopez, C. A., Fikrig, E., Hughes, M. E., and Walker, W. E. (2018). Circadian Rhythms Influence the Severity of Sepsis in Mice via a TLR2-Dependent, Leukocyte-Intrinsic Mechanism. J. Immunol. 201 (1), 193201. doi: 10.4049/jimmunol.1701677

Hemmeryckx, B., Frederix, L., and Lijnen, H. R. (2019). Deficiency of Bmal1 Disrupts the Diurnal Rhythm of Haemostasis. Exp. Gerontol. 118, 1-8. doi: 10.1016/j.exger.2018.12.017

Hofer, S., Bopp, C., Hoerner, C., Plaschke, K., Faden, R. M., Martin, E., et al. (2008). Injury of the Blood Brain Barrier and Up-Regulation of Icam-1 in
Polymicrobial Sepsis. J. Surg. Res. 146 (2), 276-281. doi: 10.1016/ j.jss.2007.07.021

Hrushesky, W. J., Langevin, T., Kim, Y. J., and Wood, P. A. (1994). Circadian Dynamics of Tumor Necrosis Factor Alpha (Cachectin) Lethality. J. Exp. Med. 180 (3), 1059-1065. doi: $10.1084 /$ jem.180.3.1059

Huang, M., Cai, S., and Su, J. (2019). The Pathogenesis of Sepsis and Potential Therapeutic Targets. Int. J. Mol. Sci. 20 (21), 444-449. doi: 10.3390/ ijms 20215376

Jacob, H., Curtis, A. M., and Kearney, C. J. (2020). Therapeutics on the Clock: Circadian Medicine in the Treatment of Chronic Inflammatory Diseases. Biochem. Pharmacol. 182, 114254. doi: 10.1016/j.bcp.2020.114254

Kasahara, E., and Inoue, M. (2015). Cross-Talk Between HPA-Axis-Increased Glucocorticoids and Mitochondrial Stress Determines Immune Responses and Clinical Manifestations of Patients With Sepsis. Redox Rep. 20 (1), 1-10. doi: 10.1179/1351000214Y.0000000107

Korneev, K. V. (2019). Mouse Models of Sepsis and Septic Shock. Mol. Biol. (Mosk) 53 (5), 799-814. doi: 10.1134/S0026898419050100

Lachmann, G., Ananthasubramaniam, B., Wunsch, V. A., Scherfig, L. M., von Haefen, C., Knaak, C., et al. (2021). Circadian Rhythms in Septic Shock Patients. Ann. Intensive Care 11 (1), 64. doi: 10.1186/s13613-021-00833-5

Leon, L. R., White, A. A., and Kluger, M. J. (1998). Role of IL-6 and TNF in Thermoregulation and Survival During Sepsis in Mice. Am. J. Physiol. 275 (1 Pt 2), R269-R277. doi: 10.1152/ajpregu.1998.275.1.R269

Lewis, A. J., Zhang, X., Griepentrog, J. E., Yuan, D., Collage, R. D., Waltz, P. K., et al. (2018). Blue Light Enhances Bacterial Clearance and Reduces Organ Injury During Sepsis. Crit. Care Med. 46 (8), e779-e787. doi: 10.1097/ CCM. 0000000000003190

Liao, Y. R., and Lin, J. Y. (2015). Quercetin Intraperitoneal Administration Ameliorates Lipopolysaccharide-Induced Systemic Inflammation in Mice. Life Sci. 137, 89-97. doi: 10.1016/j.lfs.2015.07.015

Li, X., Chen, C., Zhang, L., Cheng, X., Geng, H., Ji, Q., et al. (2021). Absence of TNF Leads to Alternative Activation in Peritoneal Macrophages in Experimental Listeria Monocytogenes Infection. Immunol. Invest. 50, 1-18. doi: $10.1080 / 08820139.2021 .1902346$

Li, J., Liu, L., Zhou, X., Lu, X., Liu, X., Li, G., et al. (2020). Melatonin Attenuates Sepsis-Induced Acute Lung Injury Through Improvement of Epithelial Sodium Channel-Mediated Alveolar Fluid Clearance Via Activation of SIRT1/SGK1/ Nedd4-2 Signaling Pathway. Front. Pharmacol. 11, 590652. doi: 10.3389/ fphar.2020.590652

Liu, J., Malkani, G., Shi, X., Meyer, M., Cunningham-Runddles, S., Ma, X., et al. (2006). The Circadian Clock Period 2 Gene Regulates Gamma Interferon Production of NK Cells in Host Response to Lipopolysaccharide-Induced Endotoxic Shock. Infect. Immun. 74 (8), 4750-4756. doi: 10.1128/IAI.00287-06

Luan, Y. Y., Yao, Y. M., Xiao, X. Z., and Sheng, Z. Y. (2015). Insights Into the Apoptotic Death of Immune Cells in Sepsis. J. Interferon Cytokine Res. 35 (1), 17-22. doi: 10.1089/jir.2014.0069

Maas, M. B., Iwanaszko, M., Lizza, B. D., Reid, K. J., Braun, R. I., and Zee, P. C. (2020a). Circadian Gene Expression Rhythms During Critical Illness. Crit. Care Med. 48 (12), e1294-e1299. doi: 10.1097/CCM.0000000 000004697

Maas, M. B., Lizza, B. D., Kim, M., Abbott, S. M., Gendy, M., Reid, K. J., et al. (2020b). Stress-Induced Behavioral Quiescence and Abnormal Rest-Activity Rhythms During Critical Illness. Crit. Care Med. 48 (6), 862-871. doi: 10.1097/ CCM.0000000000004334

Marchant, A., Deviere, J., Byl, B., De Groote, D., Vincent, J. L., and Goldman, M. (1994). Interleukin-10 Production During Septicaemia. Lancet 343 (8899), 707-708. doi: 10.1016/s0140-6736(94)91584-9

Marie, C., Pitton, C., Fitting, C., and Cavaillon, J. M. (1996). Regulation by AntiInflammatory Cytokines (IL-4, IL-10, IL-13, TGFbeta) of Interleukin-8 Production by LPS- and/ or TNFalpha-Activated Human Polymorphonuclear Cells. Mediators Inflamm. 5 (5), 334-340. doi: 10.1155/\$0962935196000488

Marik, P. E. (2011). Glucocorticoids in Sepsis: Dissecting Facts From Fiction. Crit. Care 15 (3), 158. doi: 10.1186/cc10101

Marpegan, L., Bekinschtein, T. A., Costas, M. A., and Golombek, D. A. (2005). Circadian Responses to Endotoxin Treatment in Mice. J. Neuroimmunol. 160 (1-2), 102-109. doi: 10.1016/j.jneuroim.2004.11.003

Marpegan, L., Leone, M. J., Katz, M. E., Sobrero, P. M., Bekinstein, T. A., and Golombek, D. A. (2009). Diurnal Variation in Endotoxin-Induced Mortality in 
Mice: Correlation With Proinflammatory Factors. Chronobiol. Int. 26 (7), 1430-1442. doi: 10.3109/07420520903408358

Marra, A., McGrane, T. J., Henson, C. P., and Pandharipande, P. P. (2019). Melatonin in Critical Care. Crit. Care Clin. 35 (2), 329-340. doi: 10.1016/ j.ccc.2018.11.008

Meneses, G., Cardenas, G., Espinosa, A., Rassy, D., Perez-Osorio, I. N., Barcena, B., et al. (2019). Sepsis: Developing New Alternatives to Reduce Neuroinflammation and Attenuate Brain Injury. Ann. N. Y. Acad. Sci. 1437 (1), 43-56. doi: 10.1111/ nyas. 13985

Morrison, S. F., and Nakamura, K. (2018). Central Mechanisms for Thermoregulation. Annu. Rev. Physiol. 81, 285-308. doi: 10.1146/annurevphysiol-020518-114546

Mul Fedele, M. L., Aiello, I., Caldart, C. S., Golombek, D. A., Marpegan, L., and Paladino, N. (2020). Differential Thermoregulatory and Inflammatory Patterns in the Circadian Response to LPS-Induced Septic Shock. Front. Cell Infect. Microbiol. 10, 100. doi: 10.3389/fcimb.2020.00100

Nautiyal, K. M., McKellar, H., Silverman, A. J., and Silver, R. (2009). Mast Cells Are Necessary for the Hypothermic Response to LPS-Induced Sepsis. Am. J. Physiol. Regul. Integr. Comp. Physiol. 296 (3), R595-R602. doi: 10.1152/ ajpregu.90888.2008

Oishi, K., Koyanagi, S., and Ohkura, N. (2013). The Molecular Clock Regulates Circadian Transcription of Tissue Factor Gene. Biochem. Biophys. Res. Commun. 431 (2), 332-335. doi: 10.1016/j.bbrc.2012.12.098

Okabe, Y., and Medzhitov, R. (2014). Tissue-Specific Signals Control Reversible Program of Localization and Functional Polarization of Macrophages. Cell 157 (4), 832-844. doi: 10.1016/j.cell.2014.04.016

Oyama, Y., Iwasaka, H., Koga, H., Shingu, C., Matsumoto, S., and Noguchi, T. (2014). Uncoupling of Peripheral and Master Clock Gene Rhythms by Reversed Feeding Leads to an Exacerbated Inflammatory Response After Polymicrobial Sepsis in Mice. Shock 41 (3), 214-221. doi: 10.1097/ SHK.0000000000000094

Paladino, N., Mul Fedele, M. L., Duhart, J. M., Marpegan, L., and Golombek, D. A. (2014). Modulation of Mammalian Circadian Rhythms by Tumor Necrosis Factor-Alpha. Chronobiol. Int. 31 (5), 668-679. doi: 10.3109/07420528.2014.886588

Partch, C. L., Green, C. B., and Takahashi, J. S. (2014). Molecular Architecture of the Mammalian Circadian Clock. Trends Cell Biol. 24 (2), 90-99. doi: 10.1016/ j.tcb.2013.07.002

Pfeffer, K., Matsuyama, T., Kundig, T. M., Wakeham, A., Kishihara, K., Shahinian, A., et al. (1993). Mice Deficient for the 55 Kd Tumor Necrosis Factor Receptor Are Resistant to Endotoxic Shock, Yet Succumb to L. Monocytogenes Infection. Cell 73 (3), 457-467. doi: 10.1016/0092-8674(93)90134-C

Pollmacher, T., Mullington, J., Korth, C., Schreiber, W., Hermann, D., Orth, A., et al. (1996). Diurnal Variations in the Human Host Response to Endotoxin. J. Infect. Dis. 174 (5), 1040-1045. doi: 10.1093/infdis/174.5.1040

Ramos-Benitez, M. J., Ruiz-Jimenez, C., Rosado-Franco, J. J., Ramos-Perez, W. D., Mendez, L. B., Osuna, A., et al. (2018). Fh15 Blocks the LipopolysaccharideInduced Cytokine Storm While Modulating Peritoneal Macrophage Migration and CD38 Expression Within Spleen Macrophages in a Mouse Model of Septic Shock. mSphere 3 (6), 1-11. doi: 10.1128/mSphere.00548-18

Refinetti, R., and Menaker, M. (1992). The Circadian Rhythm of Body Temperature. Physiol. Behav. 51, 613-637. doi: 10.1016/0031-9384(92)90188-8

Sertaridou, E. N., Chouvarda, I. G., Arvanitidis, K. I., Filidou, E. K., Kolios, G. C., Pnevmatikos, I. N., et al. (2018). Melatonin and Cortisol Exhibit Different Circadian Rhythm Profiles During Septic Shock Depending on Timing of Onset: A Prospective Observational Study. Ann. Intensive Care 8 (1), 118. doi: 10.1186/s13613-018-0462-y

Shearer, W. T., Reuben, J. M., Mullington, J. M., Price, N. J., Lee, B. N., Smith, E. O., et al. (2001). Soluble TNF-Alpha Receptor 1 and IL-6 Plasma Levels in Humans Subjected to the Sleep Deprivation Model of Spaceflight. J. Allergy Clin. Immunol. 107 (1), 165-170. doi: 10.1067/mai.2001.112270

Silver, A. C., Arjona, A., Walker, W. E., and Fikrig, E. (2012). The Circadian Clock Controls Toll-Like Receptor 9-Mediated Innate and Adaptive Immunity. Immunity 36 (2), 251-261. doi: 10.1016/j.immuni.2011.12.017

Singer, M. (2016). The New Sepsis Consensus Definitions (Sepsis-3): The Good, the Not-So-Bad, and the Actually-Quite-Pretty. Intensive Care Med. 42 (12), 2027-2029. doi: 10.1007/s00134-016-4600-4
Stewart, C. R., Landseadel, J. P., Gurka, M. J., and Fairchild, K. D. (2010). Hypothermia Increases Interleukin-6 and Interleukin-10 in Juvenile Endotoxemic Mice. Pediatr. Crit. Care Med. 11 (1), 109-116. doi: 10.1097/PCC.0b013e3181b01042

van der Poll, T., and Opal, S. M. (2008). Host-Pathogen Interactions in Sepsis. Lancet Infect. Dis. 8 (1), 32-43. doi: 10.1016/S1473-3099(07)70265-7

van der Poll, T., van de Veerdonk, F. L., Scicluna, B. P., and Netea, M. G. (2017). The Immunopathology of Sepsis and Potential Therapeutic Targets. Nat. Rev. Immunol. 17, 407-420. doi: 10.1038/nri.2017.36

Vincent, J. L., Marshall, J. C., Namendys-Silva, S. A., Francois, B., Martin-Loeches, I., Lipman, J., et al. (2014). Assessment of the Worldwide Burden of Critical Illness: The Intensive Care Over Nations (ICON) Audit. Lancet Respir. Med. 2 (5), 380-386. doi: 10.1016/S2213-2600(14)70061-X

Wang, C. Y., Hsieh, M. J., Hsieh, I. C., Shie, S. S., Ho, M. Y., Yeh, J. K., et al. (2016). CLOCK Modulates Survival and Acute Lung Injury in Mice With Polymicrobial Sepsis. Biochem. Biophys. Res. Commun. 478 (2), 935-941. doi: 10.1016/j.bbrc.2016.08.054

Wang, J., and Kubes, P. (2016). A Reservoir of Mature Cavity Macrophages That Can Rapidly Invade Visceral Organs to Affect Tissue Repair. Cell 165 (3), 668678. doi: 10.1016/j.cell.2016.03.009

Wang, J., Luo, Y., Wang, K., Wang, Y., Zhang, X., Teng, H., et al. (2015). ClockControlled StAR's Expression and Corticosterone Production Contribute to the Endotoxemia Immune Response. Chronobiol. Int. 32 (3), 358-367. doi: 10.3109/07420528.2014.982284

Wanner, S. P., Yoshida, K., Kulchitsky, V. A., Ivanov, A. I., Kanosue, K., and Romanovsky, A. A. (2013). Lipopolysaccharide-Induced Neuronal Activation in the Paraventricular and Dorsomedial Hypothalamus Depends on Ambient Temperature. PLoS One 8 (9), e75733. doi: 10.1371/journal.pone.0075733

Weis, S., Carlos, A. R., Moita, M. R., Singh, S., Blankenhaus, B., Cardoso, S., et al. (2017). Metabolic Adaptation Establishes Disease Tolerance to Sepsis. Cell 169 (7), 1263-1275.e1214. doi: 10.1016/j.cell.2017.05.031

Xu, L., Zhang, W., Kwak, M., Zhang, L., Lee, P. C. W., and Jin, J. O. (2019). Protective Effect of Melatonin Against Polymicrobial Sepsis Is Mediated by the Anti-Bacterial Effect of Neutrophils. Front. Immunol. 10, 1371. doi: 10.3389/ fimmu.2019.01371

Youm, Y. H., Nguyen, K. Y., Grant, R. W., Goldberg, E. L., Bodogai, M., Kim, D., et al. (2015). The Ketone Metabolite Beta-Hydroxybutyrate Blocks NLRP3 Inflammasome-Mediated Inflammatory Disease. Nat. Med. 21 (3), 263-269. doi: $10.1038 / \mathrm{nm} .3804$

Yuan, M., Li, D., An, M., Li, Q., Zhang, L., and Wang, G. (2017). Rediscovering Peritoneal Macrophages in a Murine Endometriosis Model. Hum. Reprod. 32, 94-102. doi: 10.1093/humrep/dew274

Zhang, N., Czepielewski, R. S., Jarjour, N. N., Erlich, E. C., Esaulova, E., Saunders, B. T., et al. (2019). Expression of Factor V by Resident Macrophages Boosts Host Defense in the Peritoneal Cavity. J. Exp. Med. 216 (6), 1291-1300. doi: 10.1084/jem.20182024

Zheng, B., Larkin, D. W., Albrecht, U., Sun, Z. S., Sage, M., Eichele, G., et al. (1999). The Mper2 Gene Encodes a Functional Component of the Mammalian Circadian Clock. Nature 400, 169-173. doi: 10.1038/22118

Conflict of Interest: The authors declare that the research was conducted in the absence of any commercial or financial relationships that could be construed as a potential conflict of interest.

Publisher's Note: All claims expressed in this article are solely those of the authors and do not necessarily represent those of their affiliated organizations, or those of the publisher, the editors and the reviewers. Any product that may be evaluated in this article, or claim that may be made by its manufacturer, is not guaranteed or endorsed by the publisher.

Copyright (๑) 2021 Mul Fedele, Senna, Aiello, Golombek and Paladino. This is an open-access article distributed under the terms of the Creative Commons Attribution License (CC BY). The use, distribution or reproduction in other forums is permitted, provided the original author(s) and the copyright owner(s) are credited and that the original publication in this journal is cited, in accordance with accepted academic practice. No use, distribution or reproduction is permitted which does not comply with these terms. 